ROM2F-98/10

hep-th/9803192

\title{
A Ricci-flat metric on D-brane orbifolds
}

\author{
Koushik Ray ${ }^{* \dagger}$ \\ Dipartimento di Fisica \\ Università di Roma "Tor Vergata" \\ I.N.F.N. - Sezione di Roma "Tor Vergata" \\ Via della Ricerca Scientifica, 100133 Roma ITALY
}

\begin{abstract}
We study issues pertaining to the Ricci-flatness of metrics on orbifolds resolved by Dbranes. We find a Kähler metric on the three-dimensional orbifold $\mathbb{C}^{3} / \mathbb{Z}_{3}$, resolved by D-branes, following an approach due to Guillemin. This metric is not Ricci-flat for any finite value of the blow-up parameter. Conditions for the envisaged Ricci-flat metric for finite values of the blow-up parameter are formulated in terms of a correction to the Kähler potential. This leads to an explicit construction of a Ricci-flat Kähler metric on the resolved orbifold. The correction constitutes a part of the superspace-interaction in the corresponding gauged linear sigma-model.
\end{abstract}

\footnotetext{
*I.N.F.N. Fellow.

†E-mail: koushik@roma2.infn.it
} 
Duality symmetries in Type-I and Type-II string theories necessitate incorporating Dbranes in these theories, even though the branes may be of dimensions other than unity, and are not "strings" in general. Apart from the fact that these objects fit nicely into the conformal field theoretic framework, it looms large that D-branes will play a crucial role in our final understanding of space-time, as they can be used to probe the topology and geometry of spacetime. In fact, D0-branes are deemed to be more efficient probes of space-time than strings in that they probe distance scales shorter than the string scale [1]. These considerations have made D-branes interesting objects to study in recent times.

The world-volume theory of $n$ parallel $\mathrm{D} p$-branes is described by a supersymmetric gauge theory, namely the ten-dimensional supersymmetric Yang-Mills theory, with gauge group $U(n)$, dimensionally reduced to $(p+1)$ dimensions [2]. The moduli space of this reduced theory has been interpreted as the space-time sensed by D-branes [2]. In this sense space-time is a derived concept in the theory of D-branes. It has been found that it is possible to realize the moduli space of D-branes as orbifolds by properly truncating the reduced theory to sectors invariant under the action of some discrete group. The theory of D-branes on two(complex)-dimensional ALE-spaces has been studied in detail [3, [1. Such studies realize Krönheimer's construction of ALE-spaces in physical terms for the Kleinian subgroups of $S U(2)$ acting on the two-dimensional complex space, $\mathbb{C}^{2}$. Fundamental strings on ALE-spaces have been considered earlier [5].

More recently, it has been found that D-branes can also be used to resolve three-dimensional orbifold singularities. One can thus study the short-distance behavior of Calabi-Yau manifolds as sensed by a D-brane probe in the neighborhood of a resolved orbifold singularity. In order to study the theory of a single D-brane on $\mathbb{C}^{3} / \Gamma$, where $\Gamma$ is a discrete group of order $|\Gamma|$, one starts with $|\Gamma|$ number of D-branes on the covering space $\mathbb{C}^{3}$, arranged in the regular representation of $\Gamma$. The world-volume theory of $|\Gamma|$ D-branes on $\mathbb{C}^{3}$ is a four-dimensional gauge theory with $N=4$ supersymmetry and gauge group $U(|\Gamma|)$. The complex positions of the D-branes on $\mathbb{C}^{3}$ are given by the scalars in the theory. Restricting to the sector of the D-brane Lagrangian invariant under the discrete group yields a gauged linear sigma-model. The nexus between the two-dimensional gauged linear sigma-model and the world-volume theory of a D1-brane probe has been exploited earlier [6, 7]. The moduli space of this linear sigma-model is interpreted as the sub-stringy space-time. This moduli space can be thought of as an internal space on which D-branes move as points, with the world-volume of the D-brane lying in directions transverse to this internal space. Now, if the discrete group $\Gamma$ is chosen to be a subgroup of $S U(3)$, then the resulting theory after truncation retains $N=1$ supersymmetry. This construction has been realized for different choices of the discrete group $\Gamma$; for example, $\Gamma=\mathbb{Z}_{n}$ for $n=3,5,7,9$, 11 [8, 9] and $\Gamma=\mathbb{Z}_{2} \times \mathbb{Z}_{2}[10$, 11]. It should be mentioned that in these considerations one may start with an arbitrary number $|\Gamma|$ of D-branes on the covering space because the space one considers is non-compact. The moduli space of the D-brane can be viewed as a local description of a Calabi-Yau manifold only near an orbifold point. Further generalization of these ideas to four dimensions has also been considered [12]. It has been found that toric geometry provides a convenient language for such considerations $[8,13,14]$. In the present paper we shall concentrate on the example of $\mathbb{C}^{3} / \mathbb{Z}_{3}[\mathbb{B}]$. As mentioned above, the theory of a D-brane on $\mathbb{C}^{3} / \mathbb{Z}_{3}$ is a fourdimensional $N=1$ theory, which is equivalent to the two-dimensional $N=2$ gauged linear sigma-model [15, 16] upon dimensional reduction.

Apart from the topological properties, some geometric, alias metric, properties of these resolved orbifolds have also been considered [17]. It has been found that, starting from the flat Kähler potential on the covering space $\mathbb{C}^{3}$, and then adding the Fayet-Iliopoulos terms, 
one can derive, by proper gauging, a Kähler metric on the three-dimensional resolved orbifold $\mathbb{C}^{3} / \mathbb{Z}_{3}$. However, this metric turns out to be a non-Ricci-flat one [17, 18. It has also been found that the same prescription leads to the Eguchi-Hanson metric on the two-dimensional resolved orbifold $\mathbb{C}^{2} / \mathbb{Z}_{2}$, which is Ricci-flat. This dichotomy has been attributed to the fact that, unlike a resolved $\mathbb{C}^{2} / \mathbb{Z}_{2}$, the resolved orbifold $\mathbb{C}^{3} / \mathbb{Z}_{3}$, although Kähler, is not hyper-Kähler. In particular, it has been shown that the metric derived in this fashion for $\mathbb{C}^{3} / \mathbb{Z}_{3}$ resolved into $\mathcal{O}_{\mathbb{C P}^{2}}(-3)$ assumes the form discovered by Calabi, where $\mathcal{O}_{\mathbb{C P}^{2}}(-3)$ is looked upon as having a canonical fiber over a base manifold of constant curvature. This metric is Ricci-flat only for certain complex values of the Fayet-Iliopoulos parameters, which is forbidden both physically and mathematically.

In this paper we shall follow an alternative approach to deriving Kähler metrics on toric varieties [19]. A novel feature of this approach is that it makes it possible to derive the metric starting from the toric data alone. This approach ensues from endowing a toric variety $X$ with a symplectic structure arising from a Hamiltonian action of the torus $\mathbb{T}_{\mathbb{R}}$ on $X$ [20]. It identifies the polytope $\Delta$ corresponding to the toric variety — with $\Delta$ required to satisfy certain non-singularity and non-degeneracy conditions (in particular, the polytope $\Delta$ must be Delzant [19, 21]) - with the moment polytope of the moment map of the Hamiltonian action of the torus on $X$ by a homeomorphism $X / \mathbb{T}_{\mathbb{R}} \longrightarrow \Delta$, up to translation. This correspondence provides a prescription for obtaining a Kähler metric on the variety [19]. We shall refer to this metric as the canonical metric in the sequel. This metric is not Ricci-flat in general. However, starting from the canonical metric, one may go over to any other Kähler metric within the same Kähler class by adding a well-behaved function to the (Legendre) dual (to be defined below) of the Kähler potential [21]. We pursue this line of approach here. We first find out the canonical metric, following $[19]$, on the resolved orbifold $\mathbb{C}^{3} / \mathbb{Z}_{3}$, starting from the toric data as obtained in [8]. We find that this metric is not Ricci-flat for any finite value of the blow-up parameter. We then add a function $f$ to the (Legendre) dual Kähler potential and find out a differential equation for $f$, demanding that the new metric thus obtained is Ricci-flat. The solution of this equation leads to a Ricci-flat metric on the orbifold $\mathbb{C}^{3} / \mathbb{Z}_{3}$ resolved by a D-brane. Moreover, this additional function $f$ constitutes a part of the superspace-interaction in the linear sigma-model.

In order to fix notations, let us start with some relevant features of the present construction [19]. Let $(X, \omega)$ be an effective Hamiltonian $\mathbb{T}^{n}$-space with a Kähler form $\omega$, and let $\varphi: X \longrightarrow$ $\mathbb{R}^{n}$ denote the corresponding moment map associated to the Hamiltonian action of $\mathbb{T}^{n}$ on $X$. Let $\Delta$ denote the image of $X$ on $\mathbb{R}^{n}$ under the moment map, i.e. $\Delta=\varphi(X) \subset \mathbb{R}^{n} . \Delta$ is referred to as the moment polytope and $X_{\Delta}=X$ is the toric variety associated to $\Delta$. Conversely, one can associate a toric variety $X_{\Delta}$ with the above properties, to a Delzant polytope $\Delta$ in $\mathbb{R}^{d}$, such that $\Delta$ is the moment polytope of $X_{\Delta}$. Let us recall that the polytope $\Delta$ in $\mathbb{R}^{d}$ is called Delzant if there are $d$ edges meeting at each vertex $p$ of $\Delta$ and any edge meeting at $p$ can be given the form $p+s v_{i}$, for $0 \leq s \leq \infty$, where $\left\{v_{i}\right\}$ is a basis of $\mathbb{Z}^{d}$ [19, 21]. The moment polytope $\Delta$ can be described by a set of inequalities of the form $\left\langle y, u_{i}\right\rangle \geq \lambda_{i}, i=0,2, \cdots d-1$. Here $u_{i}$ denotes the inward-pointing normal to the $i$-th $(n-1)$-dimensional face of $\Delta$ and is a primitive element of the lattice $\mathbb{Z}^{n} \subset \mathbb{R}^{n} ;\langle$,$\rangle denotes the standard scalar product in \mathbb{R}^{n}$ and $y$ is an $n$-dimensional real vector. We can thus define a set of linear maps, $\ell_{i}: \mathbb{R}^{n} \longrightarrow \mathbb{R}$,

$$
\ell_{i}(y)=\left\langle y, u_{i}\right\rangle-\lambda_{i}, \quad i=0, \cdots d-1 .
$$

Denoting the interior of $\Delta$ by $\Delta^{\circ}, y \in \Delta^{\circ}$, if and only if $\ell_{i}(y)>0$ for all $i$. 
On the open $\mathbb{T}_{\mathbb{C}^{-o r b i t}}^{n}$ in $X_{\Delta}$, associated to a Delzant polytope $\Delta$, the Kähler form $\omega$ can be written in terms of a potential $\mathcal{F}$ as [19]:

$$
\omega=2 i \partial \bar{\partial} \mathcal{F},
$$

with

$$
\mathcal{F}=\frac{1}{2} \varphi^{\star}\left(\sum_{i=0}^{d-1} \lambda_{i} \ln \ell_{i}+\ell_{\infty}\right),
$$

where $\varphi^{\star}$ denotes the pull-back of the moment map on $\Delta$ and we have defined

$$
\ell_{\infty}=\sum_{i=0}^{d-1}\left\langle y, u_{i}\right\rangle .
$$

The Kähler form $\omega$ can be written as:

$$
\omega=\frac{i}{2} \sum_{j, k=0}^{n-1} \frac{\partial^{2} \mathcal{F}}{\partial x_{j} \partial x_{k}} d z_{j} \wedge d \bar{z}_{k} .
$$

The restriction to $\mathbb{R}^{n}=\mathfrak{R e} \mathbb{C}^{n}$ of the Kähler metric corresponding to $\omega$ is the Riemannian metric given by,

$$
d s^{2}=\sum_{j, k=0}^{n-1} \frac{\partial^{2} \mathcal{F}}{\partial x_{j} \partial x_{k}} d x_{j} d x_{k} .
$$

Now, under the Legendre transform determined by the moment map $\varphi$,

$$
y_{i}=\frac{\partial \mathcal{F}}{\partial x_{i}},
$$

the metric given by (6) is the pull-back of the metric given by,

$$
d s^{2}=\sum_{j, k=0}^{n-1} \frac{\partial^{2} \mathcal{G}}{\partial y_{j} \partial y_{k}} d y_{j} d y_{k}
$$

on $\Delta^{\circ}$, where $\mathcal{G}$ is given by:

$$
\mathcal{G}=\frac{1}{2} \sum_{k=0}^{d-1} \ell_{k}(y) \ln \ell_{k}(y) .
$$

The inverse of the Legendre transform (7) is:

$$
x_{i}=\frac{\partial \mathcal{G}}{\partial y_{i}}+r_{i}, \quad i=0, \cdots, d-1,
$$

where $r_{i}$ are constants. This means that up to a linear term in the co-ordinates $y_{i}, \mathcal{G}$ is the Kähler potential Legendre-dual to $\mathcal{F}$. Moreover, the matrix

$$
\mathcal{G}_{i j}=\frac{\partial^{2} \mathcal{G}}{\partial y_{i} \partial y_{j}},
$$


evaluated at (7), i.e. at $y_{i}=\frac{\partial \mathcal{F}}{\partial x_{i}}$, is the inverse of the matrix

$$
\mathcal{F}_{i j}=\frac{\partial^{2} \mathcal{F}}{\partial x_{i} \partial x_{j}}
$$

The Ricci-tensor for the metric (12) takes the following form:

$$
\begin{aligned}
R_{i j} & =-\frac{1}{2} \frac{\partial^{2} \ln \operatorname{det} \mathcal{F}}{\partial x_{i} \partial x_{j}} \\
& =-\frac{1}{2} \sum_{k, l=0}^{n-1} \mathcal{G}^{l j} \frac{\partial^{2} \mathcal{G}^{i k}}{\partial y_{k} \partial y_{l}}
\end{aligned}
$$

where $\mathcal{G}^{i j}$ denotes the inverse of $\mathcal{G}_{i j}$. Note that, $\mathcal{F}$ in $(13)$ refers to the matrix $\mathcal{F}_{i j}$, and not the Kähler potential, unlike other places in the paper. The Ricci-scalar for this metric is then derived by multiplying (14) with $\mathcal{G}_{i j}$, which is the inverse of the metric $\mathcal{F}_{i j}$ in the $y$ co-ordinates and is given by [21,

$$
\begin{aligned}
R & =-\frac{1}{2} \sum_{i, j=0}^{n-1} \mathcal{F}^{i j} \frac{\partial^{2} \ln \operatorname{det} \mathcal{F}}{\partial x_{i} \partial x_{j}} \\
& =-\frac{1}{2} \sum_{i, j=0}^{n-1} \frac{\partial^{2} \mathcal{G}^{i j}}{\partial y_{i} \partial y_{j}}
\end{aligned}
$$

where $\mathcal{F}^{i j}$ denotes the inverse of $\mathcal{F}_{i j}$. For our purposes, it will be convenient to use the matrix $\mathcal{G}_{i j}$ in the coordinates $y$. One can, in principle, rewrite all the relevant expressions in terms of the coordinates $x_{i}$ and the matrix $\mathcal{F}_{i j}$.

With this introduction, let us now begin the study of metric properties of the orbifold $\mathbb{C}^{3} / \mathbb{Z}_{3}$ resolved by D-branes. The description of this orbifold along with its topological properties and phase structure has been studied extensively [8, 10]. We shall not repeat the construction here. Let us start from the toric data obtained in $\mathbb{8}$ for the resolved orbifold $\mathbb{C}^{3} / \mathbb{Z}_{3}$. The toric data is specified by the charges of the components of the chiral superfields in terms of a $3 \times 4$ matrix $\widetilde{T}$ as,

$$
\widetilde{T}=\left(\begin{array}{cccc}
-1 & 1 & 0 & 0 \\
-1 & 0 & 1 & 0 \\
3 & 0 & 0 & 1
\end{array}\right)
$$

Triangulating on all points yields the blow-up of the orbifold $\mathbb{C}^{3} / Z_{3}$. Given the toric data (17), one can find out the inequalities describing the corresponding Delzant polytope [20]. For this let us consider the fan in $\mathbb{R}^{3}$, corresponding to the toric data (17) whose one-dimensional cone-generators are given by the four three-vectors constituting the four columns of $\widetilde{T}$ :

$$
\mathfrak{e}_{0}=\left(\begin{array}{c}
-1 \\
-1 \\
3
\end{array}\right), \quad \mathfrak{e}_{1}=\left(\begin{array}{l}
1 \\
0 \\
0
\end{array}\right), \quad \mathfrak{e}_{2}=\left(\begin{array}{l}
0 \\
1 \\
0
\end{array}\right), \quad \mathfrak{e}_{3}=\left(\begin{array}{l}
0 \\
0 \\
1
\end{array}\right)
$$


Thus, this case corresponds to $n=3$ and $d=4$ of the above discussion. The Kernel of the matrix $\widetilde{T}$ is

$$
\operatorname{Ker} \widetilde{T}=\left(\begin{array}{c}
1 \\
1 \\
1 \\
-3
\end{array}\right)
$$

Hence, the Kähler cone of the variety $X_{\Delta}$ associated to $\widetilde{T}$ is given by

$$
\xi \equiv a_{0}+a_{1}+a_{2}-3 a_{3}>0
$$

where we have denoted the image of the support function [13, 20] at $\mathfrak{e}_{i}$ as $-a_{i}$ for $i=0, \cdots, 3$. The moment map is given by

$$
\left|z_{0}\right|^{2}+\left|z_{1}\right|^{2}+\left|z_{2}\right|^{2}-3\left|z_{3}\right|^{3}=\xi
$$

which corresponds to the D-flatness condition of a $U(1)$ gauged linear sigma-model with four chiral superfields [8, 9, 15, 16]. In (21), $z_{i}, i=0, \cdots, 3$, denote the homogeneous coordinates on the toric variety. Let us also note that this correspondence allows us to relate the blow-up parameter $\xi$, which is proportional to the Kähler class of the variety, to the Fayet-Iliopoulos parameters of the linear sigma-model, using the charge matrix (17), and hence study the phase structure [8]. For all possible real values of the Fayet-Iliopoulos parameters, $\xi$ remains positive in concordance with (20). Physically, this means that the D-branes abstain from entering the non-geometric phases of the linear sigma-model [8,22].

Using the one-dimensional cone generators $\mathfrak{e}_{i}$, we can now write down the Delzant polytope $\Delta$ in the shifted coordinates as the one defined by the inequalities:

$$
\begin{aligned}
\xi-y_{1}-y_{2}+3 y_{3} & \geq 0, \\
y_{1} & \geq 0, \\
y_{2} & \geq 0 \\
y_{3} & \geq 0
\end{aligned}
$$

where $\xi=a_{0}+a_{1}+a_{2}-3 a_{3}$ is positive as above, and we have applied a shift of $a_{i}$ to each co-ordinate $y_{i}$, for $i=1,2,3$. The above expressions are required to be strictly positive in the interior $\Delta^{\circ}$ of $\Delta$ and equality holds in each of the above relations on the boundary of the polytope defined by two-dimensional faces. We thus have the following linear functions $\ell_{i}$, for $i=0,1,2,3$,

$$
\ell_{1}(y)=y_{1}, \quad \ell_{2}(y)=y_{2}, \quad \ell_{3}(y)=y_{3}, \quad \ell_{0}(y)=\xi-y_{1}-y_{2}+3 y_{3},
$$

whose positivity determine the Delzant polytope $\Delta$ up to shifts, corresponding to the resolved orbifold $\mathbb{C}^{3} / \mathbb{Z}_{3}$.

We can now write down the potential $\mathcal{G}$ from (26):

$$
\mathcal{G}=\frac{1}{2}\left[y_{1} \ln y_{1}+y_{2} \ln y_{2}+y_{3} \ln y_{3}+\left(\xi-y_{1}-y_{2}+3 y_{3}\right) \ln \left(\xi-y_{1}-y_{2}+3 y_{3}\right)\right]
$$


The inverse Legendre transform (10) expresses the co-ordinates $x_{i}$ in terms of the co-ordinates $y_{i}$. The relations between these dual co-ordinates can be written in the following form:

$$
\begin{aligned}
\left(\xi+3 y_{3}\right)^{3} y_{3} & =\left(1+e^{2 x_{1}}+e^{2 x_{2}}\right)^{3} e^{2 x_{3}}, \\
\frac{y_{1}}{y_{2}} & =e^{2\left(x_{1}-x_{2}\right)}, \\
\frac{y_{1}}{A} & =e^{2 x_{1}},
\end{aligned}
$$

where we have defined,

$$
A=\xi-y_{1}-y_{2}+3 y_{3}
$$

and chosen the constants $r_{i}$ as $r_{1}=r_{2}=0$ and $r_{3}=2$ for simplicity. Equations (28)-(30) can be solved explicitly to write down expressions for $x_{1}, x_{2}$ and $x_{3}$ in terms of $y_{1}, y_{2}$ and $y_{3}$. One can now calculate the matrix $\mathcal{G}_{i j}=\frac{\partial^{2} \mathcal{G}}{\partial y_{i} \partial y_{j}}$, using the potential $\mathcal{G}$ obtained in (27). It takes the form:

$$
\mathcal{G}_{i j}=\frac{1}{2 A}\left(\begin{array}{ccc}
1+A / y_{1} & 1 & -3 \\
1 & 1+A / y_{2} & -3 \\
-3 & -3 & 9+A / y_{3}
\end{array}\right)
$$

Inverting this matrix to write $\mathcal{G}^{i j}$, and then differentiating twice with respect to the co-ordinates, we can compute the Ricci-scalar according to (16) as:

$$
R=\frac{12\left(\xi^{2}+72 y_{3}^{2}\right)}{\left(\xi+12 y_{3}\right)^{3}}
$$

Thus, the metric on the variety is not Ricci-flat for any finite value of the parameter $\xi$. However, the Ricci-scalar (33) vanishes as $1 / \xi$ as $\xi$ is taken to infinity. The metric in this limit is given by

$$
\mathcal{G}^{i j}=\left(\begin{array}{ccc}
2 y_{1} & 0 & 0 \\
0 & 2 y_{2} & 0 \\
0 & 0 & 2 y_{3}
\end{array}\right)
$$

The Ricci-tensor (14) also vanishes for the metric (34). Thus, in the limit of infinite $\xi$, we have a Ricci-flat metric.

However, let us note that using the formulation of [19], one can explicitly calculate the first Chern class of the variety under consideration and check that it vanishes. For this, let us note that the Kähler class of the canonical metric can be expressed in terms of the parameters $\lambda_{i}$ as [19]:

$$
\frac{[\omega]}{2 \pi}=-\sum_{i=0}^{d-1} \lambda_{i} \alpha_{i}
$$

where $\alpha_{i}, i=0, \cdots, d-1$ are constants. One can express the first Chern class of the variety $X_{\Delta}$ as a sum of the constants $\alpha_{i}$ :

$$
\mathfrak{c}_{\mathbf{1}}\left(X_{\Delta}\right)=\sum_{i=0}^{d-1} \alpha_{i}
$$


Physically, equation (36) expresses the first Chern class of the variety as a sum of the charges of the fields in the corresponding linear sigma-model. Using the expression (20) for $\xi$, which is proportional to the Kähler class of the variety, and noting that $\lambda_{i}=-a_{i}$, when the polytope $\Delta$ is written in terms of the unshifted co-ordinates, we find that

$$
\alpha_{0}=\alpha_{1}=\alpha_{2}=1, \quad \alpha_{3}=-3,
$$

up to a constant of proportionality multiplying each of $\alpha_{i}$. Hence, by (36), the first Chern class of the resolved orbifold $\mathbb{C}^{3} / Z_{3}$ vanishes. This is not surprising in view of the fact that the action of $\mathbb{Z}_{3}$ on the chiral superfields were chosen in the beginning in such a way that the $\mathbb{Z}_{3}$ was a subgroup of $S U(3)$ [ 8 . Nonetheless, the present approach provides an explicit reconfirmation of the vanishing of the first Chern class of the resolved orbifold $\mathbb{C}^{3} / \mathbb{Z}_{3}$.

Let us now go over to explore the possibility of obtaining a Ricci-flat metric for finite values of the blow-up parameter $\xi$. For this purpose, let us note [21] that one can add a function, which is smooth on some open subset of $\mathbb{R}^{n}$ containing $\Delta$, to the potential $\mathcal{G}$, such that the Hessian of the new potential is positive definite on $\Delta^{\circ}$, and derive a new Kähler metric in the same Kähler class as the canonical one. The variety $X_{\Delta}$ endowed with the two different Kähler forms are related by a $\mathbb{T}^{n}$-equivariant symplectomorphism by virtue of the function $f$ being non-singular [21, Remark 3.1]. For example, the function one has to add to the potential $\mathcal{G}$, in order to derive the extremal Kähler metric on $\mathbb{C P}^{2} \# \mathbb{C P}^{2}$ in Calabi's form, was determined utilizing this observation [21]. Following this approach, let us as add a function $f$ to $\mathcal{G}$, and define a new potential $\widetilde{\mathcal{G}}=\mathcal{G}+\frac{1}{2} f$. Now the matrix $\widetilde{\mathcal{G}}_{i j}$ corresponding to the potential $\widetilde{\mathcal{G}}$ assumes the form

$$
\widetilde{\mathcal{G}}_{i j}=\mathcal{G}_{i j}+\frac{1}{2} \frac{\partial^{2} f}{\partial y_{i} \partial y_{j}}
$$

One can then find out the Kähler metric by inverting $\widetilde{\mathcal{G}}_{i j}$ and hence the curvature for this new metric. This will also give rise to a new $\widetilde{\mathcal{F}}$ corresponding to $\mathcal{F}$ and also new co-ordinates $\widetilde{x}$. What we propose to do next is to write down the general form of the metric for a function $f$ and then determine the function by demanding that the Ricci-tensor given by the formula (14) vanishes. This gives a differential equation for the function $f$. However, for practical purposes of obtaining a tractable equation for $f$, one needs to start with an ansätz for the function $f$. In view of the fact that the Ricci-scalar (33) is a function of $y_{3}$ alone, not depending on $y_{1}$ or $y_{2}$, let us assume that $f$ is a function of $y_{3}$ only, i.e. $f=f\left(y_{3}\right)$. The matrix $\widetilde{\mathcal{G}}_{i j}$ is then given by

$$
\widetilde{\mathcal{G}}_{i j}=\frac{1}{2 A}\left(\begin{array}{ccc}
1+A / y_{1} & 1 & -3 \\
1 & 1+A / y_{2} & -3 \\
-3 & -3 & 9+A / y_{3}+A f^{\prime \prime}
\end{array}\right)
$$

where $A=\xi-y_{1}-y_{2}+3 y_{3}$, as before and a prime denotes a differentiation with respect to $y_{3}$. The inverse of the matrix $\widetilde{\mathcal{G}}_{i j}$ is given by

$$
\widetilde{\mathcal{G}}^{i j}=\left(\begin{array}{ccc}
2 y_{1}-2 \frac{y_{1}^{2}}{F} \phi & -2 \frac{y_{1} y_{2}}{F} \phi & 6 \frac{y_{1} y_{3}}{F} \\
-2 \frac{y_{1} y_{2}}{F} \phi & 2 y_{2}-2 \frac{y_{2}^{2}}{F} \phi & 6 \frac{y_{2} y_{3}}{F} \\
6 \frac{y_{1} y_{3}}{F} & 6 \frac{y_{2} y_{3}}{F} & 2 \frac{y_{3}\left(\xi+3 y_{3}\right)}{F}
\end{array}\right),
$$


where we have defined

$$
F=\xi+12 y_{3}+\left(\xi+3 y_{3}\right) y_{3} f^{\prime \prime} \quad \text { and } \quad \phi=\frac{F-9 y_{3}}{\xi+3 y_{3}} .
$$

The determinant of the matrix $\widetilde{\mathcal{G}}_{i j}$ is $\operatorname{det} \widetilde{\mathcal{G}}_{i j}=\frac{F}{8 A y_{1} y_{2} y_{3}}$. Hence, throughout this paper we shall assume $F$ to be nowhere-vanishing in order to keep $\widetilde{\mathcal{G}}_{i j}$ non-singular. For the metric (40), the surviving terms in the different components of $R_{i j}$ as evaluated from (14) take the following form:

$$
\begin{aligned}
& -2 R_{11}=\widetilde{\mathcal{G}}^{11}\left(\frac{\partial^{2} \widetilde{\mathcal{G}}^{11}}{\partial y_{1} \partial y_{1}}+\frac{\partial^{2} \widetilde{\mathcal{G}}^{12}}{\partial y_{1} \partial y_{2}}+\frac{\partial^{2} \widetilde{\mathcal{G}}^{13}}{\partial y_{1} \partial y_{3}}\right)+\widetilde{\mathcal{G}}^{13}\left(\frac{\partial^{2} \widetilde{\mathcal{G}}^{11}}{\partial y_{1} \partial y_{3}}+\frac{\partial^{2} \widetilde{\mathcal{G}}^{12}}{\partial y_{2} \partial y_{3}}+\frac{\partial^{2} \widetilde{\mathcal{G}}^{13}}{\partial y_{3} \partial y_{3}}\right) \\
& -2 R_{12}=\widetilde{\mathcal{G}}^{12}\left(\frac{\partial^{2} \widetilde{\mathcal{G}}^{11}}{\partial y_{1} \partial y_{1}}+\frac{\partial^{2} \widetilde{\mathcal{G}}^{12}}{\partial y_{1} \partial y_{2}}+\frac{\partial^{2} \widetilde{\mathcal{G}}^{13}}{\partial y_{1} \partial y_{3}}\right)+\widetilde{\mathcal{G}}^{23}\left(\frac{\partial^{2} \widetilde{\mathcal{G}}^{11}}{\partial y_{1} \partial y_{3}}+\frac{\partial^{2} \widetilde{\mathcal{G}}^{12}}{\partial y_{2} \partial y_{3}}+\frac{\partial^{2} \widetilde{\mathcal{G}}^{13}}{\partial y_{3} \partial y_{3}}\right) \\
& -2 R_{13}=\widetilde{\mathcal{G}}^{13}\left(\frac{\partial^{2} \widetilde{\mathcal{G}}^{11}}{\partial y_{1} \partial y_{1}}+\frac{\partial^{2} \widetilde{\mathcal{G}}^{12}}{\partial y_{1} \partial y_{2}}+\frac{\partial^{2} \widetilde{\mathcal{G}}^{13}}{\partial y_{1} \partial y_{3}}\right)+\widetilde{\mathcal{G}}^{33}\left(\frac{\partial^{2} \widetilde{\mathcal{G}}^{11}}{\partial y_{1} \partial y_{3}}+\frac{\partial^{2} \widetilde{\mathcal{G}}^{12}}{\partial y_{2} \partial y_{3}}+\frac{\partial^{2} \widetilde{\mathcal{G}}^{13}}{\partial y_{3} \partial y_{3}}\right) \\
& -2 R_{22}=\widetilde{\mathcal{G}}^{22}\left(\frac{\partial^{2} \widetilde{\mathcal{G}}^{12}}{\partial y_{1} \partial y_{2}}+\frac{\partial^{2} \widetilde{\mathcal{G}}^{22}}{\partial y_{2} \partial y_{2}}+\frac{\partial^{2} \widetilde{\mathcal{G}}^{23}}{\partial y_{2} \partial y_{3}}\right)+\widetilde{\mathcal{G}}^{23}\left(\frac{\partial^{2} \widetilde{\mathcal{G}}^{12}}{\partial y_{1} \partial y_{3}}+\frac{\partial^{2} \widetilde{\mathcal{G}}^{22}}{\partial y_{2} \partial y_{3}}+\frac{\partial^{2} \widetilde{\mathcal{G}}^{23}}{\partial y_{3} \partial y_{3}}\right) \\
& -2 R_{23}=\widetilde{\mathcal{G}}^{23}\left(\frac{\partial^{2} \widetilde{\mathcal{G}}^{12}}{\partial y_{1} \partial y_{2}}+\frac{\partial^{2} \widetilde{\mathcal{G}}^{22}}{\partial y_{2} \partial y_{2}}+\frac{\partial^{2} \widetilde{\mathcal{G}}^{23}}{\partial y_{2} \partial y_{3}}\right)+\widetilde{\mathcal{G}}^{33}\left(\frac{\partial^{2} \widetilde{\mathcal{G}}^{12}}{\partial y_{1} \partial y_{3}}+\frac{\partial^{2} \widetilde{\mathcal{G}}^{22}}{\partial y_{2} \partial y_{3}}+\frac{\partial^{2} \widetilde{\mathcal{G}}^{23}}{\partial y_{3} \partial y_{3}}\right) \\
& -2 R_{33}=\widetilde{\mathcal{G}}^{33}\left(\frac{\partial^{2} \widetilde{\mathcal{G}}^{13}}{\partial y_{1} \partial y_{3}}+\frac{\partial^{2} \widetilde{\mathcal{G}}^{23}}{\partial y_{2} \partial y_{3}}+\frac{\partial^{2} \widetilde{\mathcal{G}}^{33}}{\partial y_{3} \partial y_{3}}\right) \text {. }
\end{aligned}
$$

Thus, the Ricci-tensor vanishes if the following five equations are satisfied:

$$
\begin{aligned}
& \frac{\partial^{2} \widetilde{\mathcal{G}}^{11}}{\partial y_{1} \partial y_{1}}+\frac{\partial^{2} \widetilde{\mathcal{G}}^{12}}{\partial y_{1} \partial y_{2}}+\frac{\partial^{2} \widetilde{\mathcal{G}}^{13}}{\partial y_{1} \partial y_{3}}=0 \\
& \frac{\partial^{2} \widetilde{\mathcal{G}}^{11}}{\partial y_{1} \partial y_{3}}+\frac{\partial^{2} \widetilde{\mathcal{G}}^{12}}{\partial y_{2} \partial y_{3}}+\frac{\partial^{2} \widetilde{\mathcal{G}}^{13}}{\partial y_{3} \partial y_{3}}=0 \\
& \frac{\partial^{2} \widetilde{\mathcal{G}}^{12}}{\partial y_{1} \partial y_{2}}+\frac{\partial^{2} \widetilde{\mathcal{G}}^{22}}{\partial y_{2} \partial y_{2}}+\frac{\partial^{2} \widetilde{\mathcal{G}}^{23}}{\partial y_{2} \partial y_{3}}=0 \\
& \frac{\partial^{2} \widetilde{\mathcal{G}}^{12}}{\partial y_{1} \partial y_{3}}+\frac{\partial^{2} \widetilde{\mathcal{G}}^{22}}{\partial y_{2} \partial y_{3}}+\frac{\partial^{2} \widetilde{\mathcal{G}}^{23}}{\partial y_{3} \partial y_{3}}=0 \\
& \frac{\partial^{2} \widetilde{\mathcal{G}}^{13}}{\partial y_{1} \partial y_{3}}+\frac{\partial^{2} \widetilde{\mathcal{G}}^{23}}{\partial y_{2} \partial y_{3}}+\frac{\partial^{2} \widetilde{\mathcal{G}}^{33}}{\partial y_{3} \partial y_{3}}=0 .
\end{aligned}
$$

Let us note that adding the equations (48), (50) and (52) yields the equation of vanishing Ricci-scalar, as derived from (16). Using the explicit form of the matrix (40), one can now write down the equations for $F$ corresponding to (48)-(52). The equations following from (48) and (50) are identical; those from (49) and (51) are also identical. We are thus left with three 
equations following from (48), (49) and (52). These are, respectively,

$$
\begin{aligned}
F-y_{3} F^{\prime}-\phi F & =0 \\
2 y_{3}\left(F^{\prime}\right)^{2}-\left(\phi^{\prime} F-\phi F^{\prime}\right) F-y_{3} F F^{\prime \prime}-2 F F^{\prime} & =0 \\
2 y_{3}\left(\xi+3 y_{3}\right)\left(F^{\prime}\right)^{2}-y_{3}\left(\xi+3 y_{3}\right) F F^{\prime \prime}-2\left(\xi+9 y_{3}\right) F F^{\prime}+12 F^{2} & =0 .
\end{aligned}
$$

However, these three equations are not independent. Equations (54) and (55) can be solved by using only (53). Thus, finally, we need to solve only (53) in order to determine F, i.e. the function $F$ is not over-determined by the condition of Ricci-flatness of the metric. Rewriting (53) by plugging in the expression of $\phi$ from (41), we derive the following equation,

$$
y_{3}\left(\xi+3 y_{3}\right) F^{\prime}+\left(F-\xi-12 y_{3}\right) F=0 .
$$

As a check on consistency, let us note that (56) solves the equation for vanishing Ricci-scalar, which, by (16), can be written as

$$
\begin{aligned}
y_{3}\left(\xi+3 y_{3}\right)^{2} F F^{\prime \prime} & +2\left(\xi+3 y_{3}\right)\left(\xi+12 y_{3}\right) F F^{\prime} \\
& -2 y_{3}\left(\xi+3 y_{3}\right)^{2}\left(F^{\prime}\right)^{2}+6\left(F-3 \xi-18 y_{3}\right) F^{2}=0 .
\end{aligned}
$$

We shall linearize and solve (56) for $F$. Once $F$ is determined, we can use (41) to determine $f^{\prime \prime}$, and this yields the Ricci-flat metric, using $f^{\prime \prime}$ in (40). In order to solve for the Kähler potential one has to solve for $f$. Let us point out that using the definition of $F$ from (41) in (56), we have the following relation between $F$ and $f$ :

$$
f^{\prime \prime}=-F^{\prime} / F \text {. }
$$

In order to solve (56), we divide both sides of (56) by $F$, as $F$ is nowhere-vanishing, to derive the first order linear equation,

$$
y_{3}\left(\xi+3 y_{3}\right) \chi^{\prime}+\left(\xi+12 y_{3}\right) \chi=1,
$$

where $\chi=1 / F$. Equation (59) can be solved to derive the following expression for $F$ :

$$
F=\frac{9 y_{3}\left(\xi+3 y_{3}\right)^{3}}{c+\left(\xi+3 y_{3}\right)^{3}}
$$

where $c$ is a constant of integration. This in turn yields the following expression for $f^{\prime \prime}$ :

$$
f^{\prime \prime}=\frac{9 y_{3}\left(\xi+3 y_{3}\right)^{3}-\left(\xi+12 y_{3}\right)\left[c+\left(\xi+3 y_{3}\right)^{3}\right]}{y_{3}\left(\xi+3 y_{3}\right)} .
$$

Using this in the expression (40) for $\widetilde{\mathcal{G}}^{i j}$, we have a Ricci-flat metric on the variety. Equation (61) can be integrated to solve for $f$ and hence determine $\widetilde{\mathcal{G}}$. Thus, we finally have the explicit form of a Ricci-flat metric and the Kähler potential on the variety in a neighborhood of the resolved orbifold singularity $\mathbb{C}^{3} / \mathbb{Z}_{3}$.

Finally, let us discuss the physical meaning of the function $f$. Let us recall that it is customary in the context of gauged linear sigma-models to work with the metric induced from the standard metric on $\mathbb{C P}^{n}$, in which one can embed the space described by the D-flatness condition. This metric, although Kähler, is not Ricci-flat 15. It is believed that there exists 
a unique Ricci-flat Kähler metric for large values of the Fayet-Iliopoulos parameter, $\xi$, in the present case. Moreover, this Ricci-flat metric differs from the one induced from the FubiniStudy metric on $\mathbb{C P}^{n}$ in such a way that the difference between the cohomologous Kähler forms corresponding to the two metrics is given by $-i \partial \bar{\partial} T$. The difference between the corresponding the Kähler potentials, $T$, provides the superspace interaction term in the Lagrangian of the linear sigma-model, namely,

$$
\int d^{2} x d^{4} \theta T
$$

in the notation of [15]. In the limit of infinite coupling, the linear sigma-model [15] differs from the conformal invariant non-linear sigma-model by the term (62). Therefore, in order to learn about the conformal invariant model from the linear one of [15], one needs to know the form of $T$ in $(62)$.

Now, let us recall the following results from [19]. It can be proved that the de Rham cohomology classes in $H^{2}(X, \mathbb{R})$ of the canonical Kähler form $\omega$ obtained through Guillemin's construction and the metric induced on $\mathbb{C}^{3} / \mathbb{Z}_{3}$ from the Fubini-Study metric on $\mathbb{C P}^{N}$ are the same. It can also be proved [19] that: there exists a smooth function, $Q$, on $\mathbb{R}^{n}$, such that

$$
\omega_{\mathrm{FS}}=\omega+i \partial \bar{\partial} \varphi^{\star} Q
$$

Moreover, $Q$ is unique up to an additive constant. Here $\omega_{\mathrm{FS}}$ denotes the pull-back from the Fubini-Study metric on $\mathbb{C P}^{N}$. An explicit form for $Q$ is given in [19]. The Kähler classes of the forms corresponding to $\mathcal{G}$ and $\widetilde{\mathcal{G}}$ are the same [21]. The Kähler form corresponding to $\widetilde{\mathcal{G}}$ can be written as:

$$
\widetilde{\omega}=\omega_{\mathrm{FS}}+i \partial \bar{\partial}\left(f-\varphi^{\star} Q\right) .
$$

In other words, the expression $\left(f-\varphi^{\star} Q\right)$ is the difference between the potentials corresponding to the induced Fubini-Study metric on the resolved orbifold and the Ricci-flat Kähler metric (40). Hence, in the limit of strong coupling this may be identified with $T$ in (62), providing the "irrelevant" superspace interaction term needed to drive the linear sigma-model to the conformal invariant limit [15].

To conclude, in this paper we have followed a construction due to Guillemin to derive a Kähler metric on the orbifold $\mathbb{C}^{3} / \mathbb{Z}_{3}$ resolved by D-branes, in the neighborhood of the blownup orbifold point. The metric derived following [19] is not Ricci-flat for any finite value of the blow-up parameter. Then, making use of the observation that one can add a well-behaved function to the Legendre-dual Kähler potential $\mathcal{G}$ [21], we determined a function $f$ which can be added to the canonical potential to obtain a Ricci-flat metric. In this approach one derives an explicit form of the Ricci-flat metric, for all values of the blow-up parameter. The novelty of the construction of [19] is that it allows to derive the metric on the variety and the corresponding Kähler potential starting from the toric data alone, and hence avoids the complications due to the choice of seed metric [17]. The canonical metric itself can be thought of as the seed metric in this context. Since the resolution of the orbifold $\mathbb{C}^{3} / Z_{3}$ as effected by D-branes is encoded in the toric data $\widetilde{T}$, which correspond to charges of the fields in the corresponding linear sigmamodel [8], the metric (40) with $f^{\prime \prime}$ given by (61) is the metric on the resolved orbifold sensed by D-branes in a neighborhood of the orbifold point. It will be interesting to check this result by direct world-sheet computations as suggested in [17]. 
In view of the result of Guillemin relating the Kähler potentials corresponding to the canonical metric and the metric induced on the resolved orbifold from the Fubini-Study metric on $\mathbb{C} \mathbb{P}^{N}$, by a function $Q$, we find that the function $f$, combined with $Q$, gives the superspace interaction term in the strong coupling regime of the linear gauged sigma-model, which is required to drive the linear sigma model to the non-linear one with conformal symmetry. It will be interesting to see how this formulation can be used to drive the linear sigma-model to the limit of strong coupling with a large Fayet-Iliopoulos parameter. It is expected that this formulation will shed some light on the emergence of conformal symmetry in this context.

It will be interesting to extend this formulation for other choices of ansätz for $f$. Another interesting point to note is that while Calabi's form of the metric is derived from the condition that the metric on the base of the resolution $\mathcal{O}_{\mathbb{C P}^{2}}(-3)$ has a constant determinant, it is not so in the present case. Here we demand the vanishing of the Ricci-tensor $R_{i j}$ as given by (14), which is different from demanding constant determinant on the base. It will be interesting, at least mathematically, to find out an explicit relation between the two metrics. Finally, it should be possible, by following the same line of arguments as here, to generalize this formulation for other cases, for example, $\mathbb{Z}_{n}$ and $\mathbb{Z}_{2} \times \mathbb{Z}_{2}$. We hope to return to some of these issues in near future.

Acknowledgements: I take this opportunity to thank M Bianchi and A Sagnotti for encouragement and enlightening discussions. I also thank D Polyakov for useful discussions.

\section{References}

[1] M Douglas, D Kabat, P Pouliot and S Shenker. D-brane and short distances in string theory; hep-th/9608024.

[2] E Witten. Bound states of strings and D-branes; hep-th/9510135.

[3] M Douglas and G Moore. D-branes, Quivers, and ALE instantons; hep-th/9603167.

[4] C Johnson and R Myers. Aspects of Type IIB theory on ALE spaces; hep-th/9610140.

[5] M Bianchi, F Fucito, G Rossi and M Martellini. ALE instantons in string effective theory; Nucl. Phys. B440 (1995) 129.

[6] M Douglas. Branes within branes; hep-th/9512077.

[7] M Douglas. Gauge fields and D-branes; hep-th/9604198.

[8] M Douglas, B Greene and D Morrison. Orbifold resolution by D-branes; hep-th/9704151.

[9] T Muto. D-branes on orbifolds and topology change; hep-th/9711090.

[10] B Greene. D-brane topology changing transitions; hep-th/9711124.

[11] S Mukhopadhyay and K Ray. Conifolds from D-branes; hep-th/9711131; to appear in Phys. Lett. B (1998) .

[12] K Mohri. D-branes and quotient singularities of Calabi-Yau fourfolds; hep-th/9707012. 
[13] B Greene. String theory on Calabi-Yau Manifolds; hep-th/9702155.

[14] N Leung and C Vafa. Branes and toric geometry; hep-th/9711013.

[15] E Witten. Phases of $N=2$ theories in two dimensions; hep-th/9301042, Nucl. Phys. B403 (1993) 159.

[16] $\mathrm{P}$ Aspinwall and $\mathrm{B}$ Greene. On the geometric interpretation of $N=2$ superconformal theories; Nucl. Phys. B437 (1995) 205.

[17] M Douglas and B Greene. Metrics on D-brane orbifolds; hep-th/9707214.

[18] M Douglas. D-branes and Matrix theory in curved space; hep-th/9707228.

[19] V Guillemin. Kähler structures on toric varieties; J. Diff. Geo. 40 (1994) 285.

[20] D Cox. Recent developments in toric geometry; alg-geom/9606016.

[21] M Abreu. Kähler geometry of toric varieties and extremal metrics; dg-ga/9711014.

[22] E Witten. Phase transitions in $M$ theory and F theory; hep-th/9603150, Nucl. Phys. B471 (1996) 195. 